\title{
Fab Helmet: ventilación mecánica no invasiva para pacientes con COVID-19.
}

\author{
Ana María Sandoval-Poveda ${ }^{1}$ \\ Diana Hernández-Montoya ${ }^{2}$ \\ Jorge Espitaleta-Gómez ${ }^{3}$
}

1. Universidad Estatal a Distancia (UNED), Vicerrectoría de Investigación, Laboratorio de Fabricación

Kä Träre; amsandoval@uned.ac.cr; iD https://orcid.org/0000-0001-8795-7355

2. Universidad Estatal a Distancia (UNED), Vicerrectoría de Investigación, Laboratorio de Fabricación

Kä Träre; dhernandez@uned.ac.cr; (D) https://orcid.org/0000-0002-8219-3234

3. Caja Costarricense del Seguro Social, Hospital del Trauma/Centro COVID-CEACO;

jespitaletag@grupoins.com; (D) https://orcid.org/0000-0002-0178-7242

\section{RESUMEN}

Fabricación del Fab Helmet: ventilación mecánica no invasiva para pacientes con COVID-19. Objetivo: Este artículo de divulgación pretende dar a conocer los aprendizajes obtenidos a partir de la elaboración del Fab Helmet. Método: Trabajo interdisciplinario e interinstitucional para la elaboración del dispositivo, considerando los que se importan desde otros países y que se usan en los hospitales nacionales, pero también las adaptaciones necesarias y posibles para Costa Rica y su realidad. Esto implica que tanto los materiales disponibles en el mercado nacional como el tipo de componentes de dicho dispositivo debían ser compatibles para funcionar adecuadamente en las condiciones del sistema de salud costarricense. Resultados: Construcción del prototipo adecuado para Costa Rica, replicable con materiales y mano de obra nacional; dotación al sistema médico de más de 200 Fab Helmet, se registra la documentación y la sistematización completa del proceso; obtención del permiso de uso por emergencia para el dispositivo, del permiso de funcionamiento para la elaboración de dispositivos médicos y odontológicos en los espacios del Laboratorio de fabricación de la UNED y del único registro sanitario de un Equipo y Material Biomédico categoría 2 proveniente de una universidad pública. Conclusiones: El proceso de trabajo realizado dejó una serie de aprendizajes tanto para el equipo de la UNED como para los médicos relacionados con el proyecto, estos se muestran en este artículo de divulgación.

Palabras clave: diseño, fabricación digital, prototipo, interfaz, Fab Lab, salud.

\section{ABSTRACT}

Manufacture of the Fab Helmet: Non-Invasive Mechanical Ventilation for Patients with COVID-19. Objective: This article aims to report the lessons learned from the development of the Fab Helmet. Method: Interdisciplinary and interinstitutional work for the elaboration of the device, considering those imported from other countries and used in national hospitals, but also the necessary and possible adaptations for Costa Rica and its reality. This implies that both materials available in the national market and the type of components of the device had to be compatible to work adequately in the conditions of Costa Rican health system. Results: Construction of the prototype suitable for Costa Rica, replicable with national materials and staff; supplying the medical system with more than 200 Fab Helmets, documentation and complete systematization of the process; obtaining the emergency 
permission for using the device, the operating permission for manufacturing medical and dental devices in UNED's Fabrication Laboratory and the only sanitary registration for category 2 Biomedical Equipment and Material given to a public university. Conclusions: Work process carried out left a series of lessons learned for both UNED team and the doctors involved in the project.

Key words: design, digital fabrication, prototype, interface, Fab Lab, health.

\section{INTRODUCCIÓN}

El proyecto "Fab Helmet: ventilación mecánica no invasiva para pacientes con COVID-19" se planteó en el primer cuatrimestre de 2020 con el objetivo principal de proveer, al área médica nacional, de un mecanismo de respiración asistida no invasiva; de manera que se colaborara con el avance del país en la lucha contra la pandemia COVID-19.

Por nacer de la pandemia, no se había realizado investigación específica de los dispositivos de ventilación mecánica no invasiva elaborados con métodos y equipos de fabricación digital. No se trabajó a partir de cero: se tomó en cuenta la experiencia del Fab Lab Kä Träre en el desarrollo de elementos para el área médica y las relaciones existentes con otros laboratorios de fabricación y con diferentes personas, instituciones y agrupaciones del Sector Salud que ya habían desarrollado este tipo de recursos o que, por encontrarse en pandemia, estaban realizando estos mismos procesos. Para que el proyecto sea más claro se definen dos conceptos.

Un laboratorio de fabricación (fab lab, por su nombre en inglés) es un "lugar para el aprendizaje y la innovación. Estos espacios brindan acceso al medio $[\cdots]$, las habilidades, los materiales y la tecnología avanzada para permitir que cualquier persona en cualquier lugar pueda hacer (casi) cualquier cosa" (Fab Foundation, s.f., párr. 1). La Universidad Estatal a Distancia (UNED) de Costa Rica, cuenta con un fab lab desde 2014, denominado Laboratorio de Fabricación Kä Träre (Fab Lab Kä Träre), el cual es un espacio abierto que trabaja con tecnologías de fabricación digital.

La ventilación mecánica no invasiva "es una forma de sostén ventilatorio administrado sin obligación de intubación endotraqueal" (Díaz, 2016, p. 5). En otras palabras, es una forma de administrar oxígeno artificialmente, pero sin intubar a la persona. Además, como lo indican Lucchini, Giani, Isgrò, Rona y Foti (2020), se sugiere este tipo de sistema de ventilación no invasiva para los pacientes que necesitan presión positiva continua en las vías respiratorias (CPAP), pues con esto se obtiene la asistencia deseada y una mayor comodidad para el paciente.

El proyecto buscó crear un dispositivo de ventilación mecánica no invasiva con tecnologías de fabricación digital pertinente a la realidad de Costa Rica, con materiales y mano de obra ubicadas en el territorio nacional. La propuesta de crear este tipo de dispositivo nació de la crisis sanitaria y de la necesidad de aportar al país, según las indicaciones de la Organización Mundial de la Salud (OMS), la cual indicó que los países debían "adoptar un enfoque basado en la participación de todo el gobierno y de toda la sociedad, en torno a una estrategia integral dirigida a prevenir las infecciones, salvar vidas y reducir al mínimo sus efectos” (Organización Mundial de la Salud, 2020, párr. 19).

La situación sanitaria mundial es bien conocida. Se anota que, en los picos más altos de contagio, la capacidad hospitalaria costarricense es sobrepasada por la demanda y bordea muy peligrosamente el punto del colapso de los servicios. La propuesta es, por lo tanto, un esfuerzo para paliar la situación y alivianar la tarea titánica que el personal médico y hospitalario enfrentan día con día. Por esto, se buscó trabajar en un dispositivo que pudiera ser usado a lo largo y ancho del país, solo con el ajuste de válvulas necesario para que fuera de carácter universal. 
Los dispositivos de ventilación mecánica no invasiva ya existían antes de la pandemia, por lo que este dispositivo no es una creación nacional, sino que se basa en el casco de la marca de StarMed (Intersurgical Ltd., 2019), que es el más conocido y utilizado internacionalmente y que es el que se importa en el país para su uso en los hospitales. Es a partir de los estudios realizados entre 2012 y 2015 por la epidemia del SARS (Patel, Wolfe, Pohlman, Hall y Kress, 2016), que sus adaptaciones se habían trabajado con éxito en otros países, como es el caso de Argentina (Pérez, 2020). De hecho, es a partir de las investigaciones y prototipos de este tipo de dispositivos que surgen iniciativas como Helmet Based Ventilation.com que, potenciadas por la pandemia, venían desarrollando y considerando la necesidad de contar con estos dispositivos médicos en todo el mundo.

Es necesario aclarar que este dispositivo no se desarrolló dentro de un ensayo clínico, ya que los protocolos para la aprobación de un medicamento o dispositivo están diseñados para ser llevados a cabo en un período extenso; por lo que "es una vía potencial para que un paciente con una afección grave o enfermedad que ponga en peligro su vida, pueda acceder a un producto médico en investigación (fármaco, medicamento biológico o dispositivo médico) para su tratamiento" (FDA, 2020, párr. 1). Esto se da cuando no se tienen opciones terapéuticas alternativas comparables o satisfactorias.

Al contar con un Laboratorio de Fabricación, las herramientas para construir el dispositivo deseado y la experiencia para realizar pruebas y lograr los resultados, la UNED se planteó el reto de trabajar con especialistas en los temas, para hacer realidad la elaboración de este prototipo en el menor tiempo posible, pero atendiendo de manera paralela todo lo correspondiente no solo a su elaboración, sino a la sistematización del proceso y cumplimiento de las regulaciones que a nivel nacional se establecen para su formalización e inscripción. El equipo de investigación estuvo integrado por Farith Tabash, Esteban Castro, Diana Hernández, Ana María Sandoval, Jorge Espitaleta, Nicolás Bautista y José Miguel Ramírez.

Para conseguir el objetivo, se establecieron las siguientes metas intermedias:

1. Establecer las características principales de los mecanismos de respiración asistida no invasiva que deben considerarse para la construcción de un prototipo funcional.

2. Determinar los materiales más adecuados para la construcción de una escafandra para usos médicos requeridos para el tratamiento de personas enfermas de COVID-19.

3. Construir diferentes prototipos según los ajustes que las valoraciones del personal médico señalen para la mejora de las diferentes propuestas.

4. Valorar, por medio del criterio del personal médico y del área de salud; los diferentes prototipos que se generen.

5. Construir un conjunto de prototipos funcionales y finales para ser usados en los servicios médicos nacionales; de forma que para su fabricación se requiera la menor cantidad posible de materiales o insumos importados.

\section{MATERIALES Y MÉTODOS}

Luego de la revisión bibliográfica, se procedió a la fase de elaboración del prototipo. Se optó por usar de base el diseño de StarMed, pero con las adaptaciones en formas y materiales que ya había trabajado el Ing. Roberto Solano, con dos médicos intensivistas. La elaboración de este nuevo prototipo estuvo a cargo del arquitecto Castro y el ingeniero en diseño industrial Tabash. Todas sus propuestas fueron valoradas con los especialistas médicos del proyecto Dr. Espitaleta, Dr. Cubero y Dr. Ramírez y por el maker Sr. Bautista; quienes valoraban los aportes y mejoraban lo propuesto. Además, el señor Bautista, se encargó de crear alianzas y apoyos entre entidades: la UNED con el Centro Especializado de Atención de Pacientes con COVID-19 (CEACO), o especialistas con diseñadores de España e Italia que tenían en abierto válvulas, necesarias para el funcionamiento del 
casco, para imprimirlas en 3D y adaptarlas a las condiciones de los centros médicos nacionales, entre otros.

Las adaptaciones mencionadas van desde los materiales disponibles en Costa Rica, hasta las conexiones a equipos instalados dentro de los hospitales costarricenses. El resultado es un dispositivo con todos los aditamentos necesarios para ser utilizado con una persona enferma de COVID-19 que requiera este tipo de mecanismo de ventilación; en la Fig. 1 puede verse el dispositivo y sus partes.

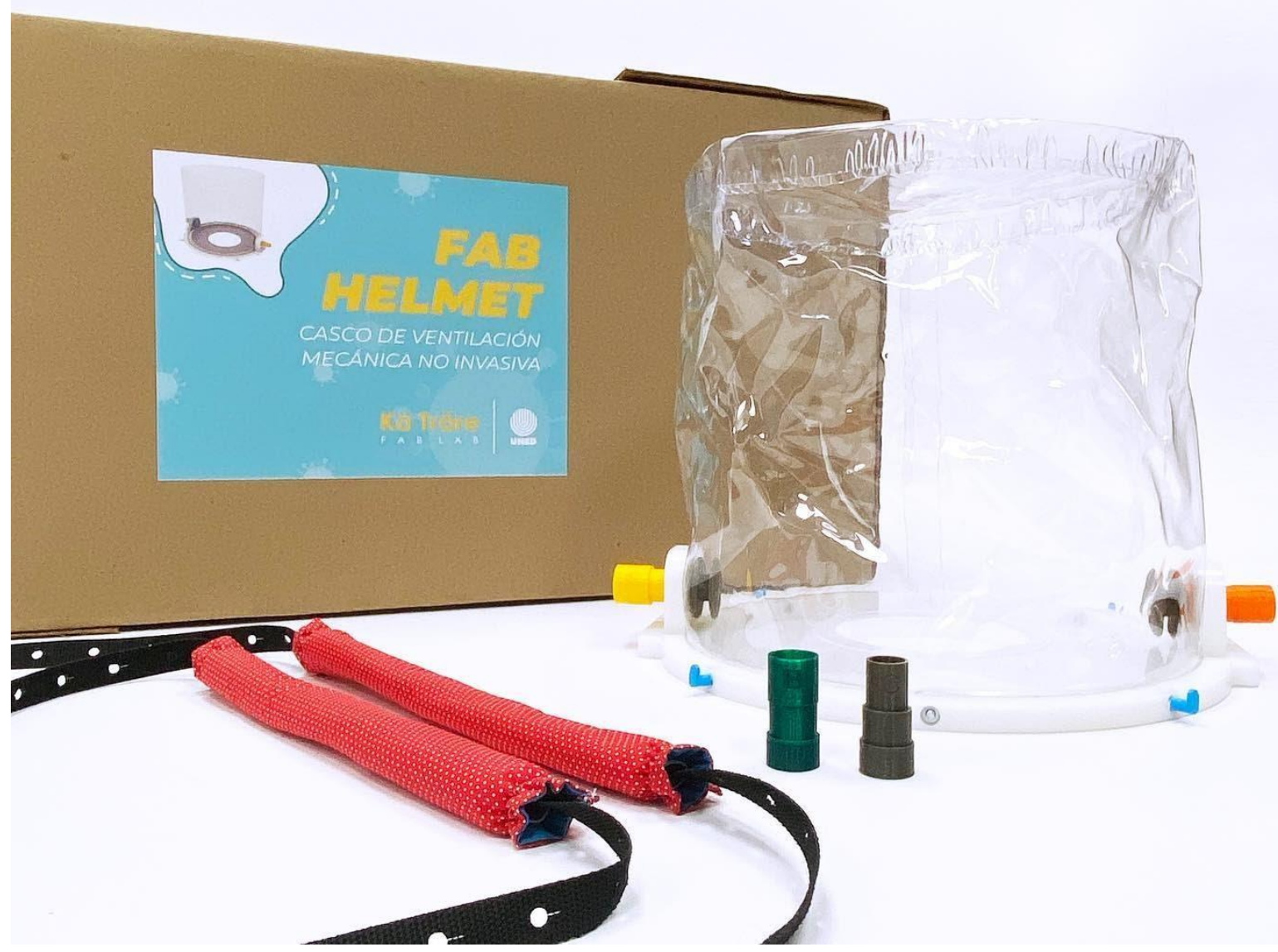

Fig. 1. Fab Helmet, listo para ser empacado. Derecha: casco de plástico libre de fisuras y conexiones para colocar válvulas (estas adelante del casco) y patillas en su base inferior. Las válvulas y conexiones fueron impresas en 3D. Tiras de sujeción recubiertas con almohadillas de tela de algodón (extremo izquierdo inferior de la imagen).

La participación como investigador del Dr. Espitaleta, facilitó que se contara con posibilidades para la realización de pruebas del primer prototipo del dispositivo en el CEACO. Esta etapa estuvo compuesta por repeticiones de: elaboración de prototipo, prueba y ajuste; con la meta de que se evidencie la seguridad y la eficacia del dispositivo; según la Ley 9234 de 2014 (artículo 2).

Todas las pruebas llevadas a cabo con los diferentes dispositivos se hicieron usando las interfaces con personas funcionarias de salud, nunca con pacientes. En el caso de la validación de la capacidad de las válvulas Venturi y PEEP, impresas en 3D, se trabajó con las empresas privadas Eleinmsa y Camérica que hicieron las mediciones y calibraciones correspondientes y aprobaron su capacidad. 
Además, se contó con aportes de personas y empresas, que se detallan en la sección de agradecimientos.

Con el prototipo listo, se procedió a elaborar varias unidades para que personas médicas intensivistas tuvieran la posibilidad de probarlos y valorar su uso. Nuevamente, fue probado con personas sanas. A partir de su visto bueno, se procedió a la primera meta: la elaboración de 100 cascos. A partir de gestiones de la Rectoría de la UNED, esta cantidad creció y se llegó a un nuevo número meta: 200 cascos, 100 para entregar en 2020 y 100 más a inicios de 2021.

Es necesario señalar que todo el trabajo manual de uso de maquinaria específica de fabricación digital y demás, no fue subcontratado. Se llevó a cabo con las personas disponibles del Laboratorio $y$, en el caso de algunos elementos particulares, con la colaboración de personas voluntarias. Solo se compraron elementos que formarían parte de los Fab Helmet, pero el armado se hizo de forma manual y por equipos de trabajo a lo interno de UNED. Paralelamente, se trabajó en otros detalles, como la redacción de los documentos de registro del trabajo, la elaboración del Manual de Uso, gestiones administrativas de compras y presupuesto, registro sanitario del dispositivo y el proceso para obtener un permiso de elaboración de equipo y material biomédico. Es necesario destacar que todo el financiamiento provino de la UNED.

Ética, conflicto de intereses y declaración de financiamiento: las personas autoras declaran haber cumplido con todos los requisitos éticos y legales pertinentes, tanto durante el estudio como en el manuscrito; también declaran que no hay conflictos de interés de ningún tipo, y que todas las fuentes financieras se detallan plena y claramente en la sección de agradecimientos. Asimismo, están de acuerdo con la versión editada final del documento. El respectivo documento legal firmado se encuentra en los archivos de la revista, en la UNED.

\section{RESULTADOS}

Paralelamente a la obtención del prototipo, se presentó a diferentes autoridades, tanto para que lo conocieran como para que gestionaran su uso en las instituciones de salud del país. Aparte de personas investigadoras del proyecto, en algunas de estas presentaciones se contó incluso con la presencia del Rector de la UNED, MBA. Rodrigo Arias. Algunos de los entes a los que se dio a conocer el proyecto son los siguientes: Colegio de Médicos y Cirujanos de Costa Rica (CMC), Red de Servicios de Salud del Instituto Nacional de Seguros (INS), Ministerio de Salud Pública de Costa Rica (MS) y Caja Costarricense del Seguro Social (CCSS). Se solicitaron audiencias con las personas encargadas de dichas entidades. De esto, se lograron dos acuerdos: MS-DM-8550- 2020 y MS-DM-8567- 2020, ambos son oficios del Ministro de Salud, Dr. Daniel Salas, dirigidos al director de la CCSS, Dr. Román Macaya, en los cuales se aprobó el uso del Fab Helmet, en caso necesario, aclarándose que el proyecto Fab Helmet no es una investigación clínica. Como cierre, se acordó la donación de los cascos.

Internamente, un resultado del ámbito administrativo fue establecer un proceso de compra a crédito que la Universidad no tenía entre sus procedimientos, aportándose un adicional no esperado que beneficia a la comunidad universitaria con una solución viable ante situaciones de emergencia.

Por otro lado, a partir de este proyecto, durante el segundo semestre de 2020, la institución como tal obtuvo el permiso para el trabajo con materiales biomédicos. El primer paso fue cumplir con las pautas que el Ministerio de Salud establece para los espacios en los que se elaboren Equipos y 
Materiales Biomédicos (EMB). Por su parte, la UNED contaba con permiso sanitario de funcionamiento para centro educativo con laboratorios, pero esto no incluía la elaboración de equipos y materiales de tipo biomédico u odontológico. Por ello, se procedió a tramitar dicha autorización, la cual está vigente hasta 2025 para el Centro Universitario de San José, donde se encuentra la segunda sede del Laboratorio de Fabricación.

Otro resultado, que era de interés real de la administración de la Universidad de que se realizara, fue la obtención del Registro Sanitario del Fab Helmet (EMB-CR-20-03307). Este trámite se realizó en diciembre de 2020 y estuvo a cargo de la investigadora Diana Hernández. Este registro fue solicitado por el Ministerio de Salud, siendo actualmente el primer y único registro obtenido por una universidad estatal de un "EMB tipo 2". Esta clasificación fue realizada por autoridades del Ministerio de Salud, de manera que el Fab Helmet, se reconoce como un dispositivo EMB2, a partir de ese momento.

Como último resultado, se detalla la entrega de los cascos. Inicialmente se proyectó que fuera directamente a la CCSS, pero no fue posible porque hasta mucho tiempo después de entregados los cascos a los entes de salud, fue remitido el procedimiento para el acto. Por lo que se procedió a entregarlo al Colegio de Médicos y Cirujanos de Costa Rica, entidad que se encargó de la distribución de los Fab Helmet. En total, la UNED entregó los cascos de la siguiente manera: 20 dispositivos a las personas del área médica encargada de hacer pruebas, 40 al Centro Especializado de Atención de Pacientes con COVID-19 (CEACO), 40 al Hospital Dr. Rafael Ángel Calderón Guardia, 100 más al Colegio de Médicos y Cirujanos de Costa Rica y 6 al Hospital de Upala. En total, se elaboraron 206 Fab Helmet, a partir del trabajo comprometido de personas ciudadanas-costarricenses y con materiales disponibles en el territorio nacional. Los 6 cascos adicionales se hicieron los materiales adquiridos restantes.

\section{DISCUSIÓN}

Al ser este un artículo de carácter divulgativo, la discusión se centra en los aprendizajes obtenidos por el equipo de investigación. Para determinar estos aprendizajes se consultó a todos los investigadores involucrados por tema y se registró la información.

Trabajo en conjunto. Cuando se cuenta con personas especialistas en diferentes áreas del saber, los proyectos se enriquecen, reciben influencias disímiles que generan soluciones distintas constantemente. Además, contar con personas expertas en tópicos específicos, como los trámites administrativos, la operación de ciertos equipos o en temas menos novedosos como la costura, todos contribuyen para que el prototipo elaborado se adapte mejor a las necesidades determinadas previamente. Adicionalmente, es necesario acotar que los equipos interdisciplinarios deben caracterizarse por el afán innovador, ya que solo de esa manera pueden impactar positivamente otras áreas, aportando así al bien común.

Equipos y materiales. Al presentarse la limitante de tener que usar solo los equipos que se tenían y materiales de disposición en el territorio nacional, fue preciso aprender acerca de ambas condicionantes. Principalmente, porque su uso no sería solo como prototipo; sino como un dispositivo de uso real y eficaz. Por otra parte, los nuevos usos para materiales conocidos o nuevas formas de trabajar los mismos materiales son aprendizajes de este proceso, que se enriquece al considerar que el uso de Recursos Educativos Abiertos permite ampliar opciones y minimizar tiempos, pues se realizó un trabajo de calidad, que, puesto a disposición del público, genera 
progreso para el país.

Apoyo al Sector Salud. Aunque todos los aprendizajes del proceso coadyuvan a avanzar, este en particular se ha dado a pasos agigantados. El Fab Lab de la UNED tenía previa experiencia en trabajos relacionados con el área médica, pero con la pandemia, esta área creció muy rápidamente. Antes del Fab Helmet, se trabajó en una careta protectora para cirujanos, videolaringólogos y también en sujeta mascarillas para personal médico. Todos estos productos permitieron valorar, con más exactitud, la necesidad de la eficiencia y la exactitud. Diseñar para el Sector Salud era un reto y se cumplió a cabalidad. Las pruebas de los productos obtenidos así lo indican y el uso dado a tales dispositivos, lo ratifica. No obstante, debe enfatizarse que las observaciones y aportes de personas especialistas en salud son indispensables para la obtención de buenos resultados. Adicionalmente, fue evidente que la fabricación digital tiene mucho que aportar al Sector Salud. A partir de ese aprendizaje, la UNED trabaja junto con la Universidad Anáhuac de Querétaro en un curso abierto y masivo para esta población que le permita sacar mayor provecho de tales tecnologías. El trabajo con el área de la salud es minucioso, detallado y exacto, razón por la cual siempre debe contarse con un buen equipo de apoyo y tener la flexibilidad de adecuar materiales y procesos para lograr la meta planteada. De forma sinérgica, la UNED puede aprovechar esta coyuntura para que el Laboratorio de Fabricación Kä Träre se integre con proyectos de graduación de estudiantes de carreras relacionadas con la tecnología, procesos productivos y la salud, potenciando así la experiencia adquirida.

Depuración de procesos. Todas las personas que se involucran en un proyecto tienen algo que aportar. Cuando esto se gestiona adecuadamente, los resultados pueden ser asombrosos. Cuanto más se depure un proceso, más beneficios hay para otros futuros proyectos, pues no se requerirá de nuevos procesos de aprendizaje, "desde cero". Queda un aprendizaje pendiente que debe ser elevado a las autoridades: los procesos de innovación requieren que las relaciones entre el gobierno y las empresas con potencial de aportar a un área particular tengan convenios o vías de comunicación abiertas. Sin colaboración y pasión no hay innovación, entendida esta como la integración y sostenibilidad real de las ideas y los procesos creativos en las acciones cotidianas de las empresas, las instituciones y los espacios.

\section{CONCLUSIONES}

A partir de los aprendizajes realizados, es posible establecer conclusiones y recomendaciones respecto al proceso, para futuros proyectos, futuras personas investigadoras que se dediquen a temas similares y para la institución misma.

El primer detalle es referente al trabajo en equipo. Es evidente que, mientras más aportes pertinentes se tengan a un mismo producto, mejores resultados se obtendrán. Pero no es posible desestimar los aportes que hacen personas de áreas ajenas al tema en investigación, pues muchas generan buenas ideas y apoyos de dicho personal. Es así como se está de acuerdo con diferentes autores que afirman que "Un buen trabajo en equipo puede derivar en un mejor servicio $y$, en consecuencia, en clientes satisfechos" (Cervantes, Muñoz e Inda, 2020, p. 8).

Respecto a los equipos y materiales, también vale la pena rescatar que durante este proyecto se aprendió mucho sobre las potencialidades de algunos equipos que antes solo se habían usado para trabajos básicos. Lo más importante no son los materiales ni el equipo, es el recurso humano. El equipo de trabajo concuerda con García (2016), quien afirma lo siguiente: "Sin duda, la mejor forma 
de conocer el futuro es participando de su creación" (p. 65). Solo al proponerse proyectos diferentes e innovadores, el ser humano es capaz de encontrar sus verdaderos límites.

En cuanto al apoyo al Sector Salud, se tiene que la necesidad expresada por la OMS no ha cambiado. Cada país debe buscar la manera más adecuada de solventar sus necesidades desde el ambiente interno. En la Declaración final acerca de la novena reunión del Comité de Emergencia del Reglamento Sanitario Internacional (2005) sobre la pandemia de enfermedad por coronavirus (COVID-19), se indica que: "Es necesario seguir investigando los aspectos de «Una sola salud» del SARS-CoV-2, las herramientas y los dispositivos, y las vacunas, las pruebas diagnósticas y los tratamientos de próxima generación para el control a largo plazo de la pandemia" (Organización Mundial de la Salud, 2021, párr. 19); por lo que los aportes a esta área del saber deben seguir dándose.

Por lo pronto, el proyecto del Fab Helmet forma parte de la investigación realizada por la Organización para la Cooperación y el Desarrollo Económicos (OCDE), por medio de su Directorate for Science, Technology and Innovation Committee for Scientific and Technological Policy, titulada Cross-country COVID-19 co-creation initiatives: Case study summaries. Es de esta manera que se reconoce el trabajo realizado y, también, las posibilidades enormes que, como país, se tiene para contribuir en situaciones de emergencia o cotidianas a solventar problemas, para atender necesidades y experimentar y crear, con el objetivo de vivir mejor.

\section{AGRADECIMIENTOS}

Se agradece profundamente a la UNED por apoyar el proyecto y brindar el financiamiento necesario para hacerlo realidad. Muchas instancias universitarias dieron apoyo a la propuesta, como es el caso de Rectoría, Vicerrectoría de Investigación, PROLAB, Vicerrectoría Ejecutiva, Unidad de Transportes, Unidad de Mantenimiento, Oficina de Distribución y Ventas, Oficina Financiera, Control de Presupuesto, Presupuesto, Tesorería y Fundación de la Universidad Estatal a Distancia para el Desarrollo y Promoción de la Educación a Distancia (FUNDEPREDI).

Adicionalmente, personas y entidades externas también participaron del proceso, cobrando los materiales, pero donando su tiempo y experiencia. Tal es el caso del señor Alberto Montes (impresión componentes por medio de impresoras 3D) y el del señor Jonathan Torres (elaboración de las partes inferiores del Fab Helmet). También, la empresa CASS-Central American Silicone Suppliers, en particular el señor Jaime Mejía Botero, colaboró con la apertura de un nuevo proceso de producción para crear los cuellos de silicón y hacer el estudio de composición. Se le compró el silicón, pero la empresa asumió los procesos.

Por último, se contó con un equipo de voluntarias en costura, convocadas en abierto por la página de Facebook del Fab Lab. Se recibieron más de 40 ofertas y se trabajó con un grupo en la elaboración de almohadillas para los sujetadores. 


\section{BIBLIOGRAFÍA}

Asamblea Legislativa de la República de Costa Rica. (2014). Ley N9234. Ley Reguladora de Investigación Biomédica. Recuperado de http://www.pgrweb.go.cr/scij/Busqueda/Normativa/Normas/nrm texto completo.aspx ?param1=NRTC\&nValor1=1\&nValor2=77070\&nValor3=96424\&strTipM=TC

Cervantes, G., Muñoz, G., \& Inda, A. D. (2020). El trabajo en equipo y su efecto en la calidad del servicio a clientes. Revista Espacios, 41(14), 27-37. Recuperado de http://ww.revistaespacios.com/a20v41n14/a20v41n14p27.pdf

Díaz, L. B. (2016). Efectividad de la musicoterapia en el nivel de ansiedad en pacientes con ventilación mecánica no invasiva en una Unidad de Cuidados Intensivos. (Tesis para Especialista en Enfermería en Cuidados Intensivos). Universidad Peruana Cayetano Heredia, Huaraz, Perú. Recuperado de https://repositorio.upch.edu.pe/bitstream/handle/20.500.12866/7664/Efectividad Diaz Rimac Liz.pdf?sequence=1\&isAllowed $=y$

Fab Foundation. (s. f.). What is a Fab Lab? Recuperado de https://fabfoundation.org/gettingstarted/\#fablabs-full

García, C. (2016). (Casi) Todo por hacer. Una mirada social y educativa sobre los Fab Lab y el movimiento maker. Madrid: Fundación Orange.

Intersurgical Ltd. (2019). Cascos Respiratorios StarMed de Intersurgical. Recuperado de http://www.elhospital.com/temas/Cascos-respiratorios-StarMed-deIntersurgical+132370

Lucchini, A., Giani, M., Isgrò, S., Rona, R. \& Foti, G. (2020). The "helmet bundle" in COVID-19 patients undergoing non invasive ventilation. Intensive and Critical Care Nursing, 58, 102859. https://doi.org/10.1016/j.iccn.2020.102859

Organización Mundial de la Salud. (2020, 11 de marzo). Alocución de apertura del director general de la OMS en la rueda de prensa sobre la COVID-19 celebrada el 11 de marzo de 2020. Recuperado de https://www.who.int/es/dg/speeches/detail/who-director-general-sopening-remarks-at-the-media-briefing-on-covid-19---11-march-2020

Organización Mundial de la Salud. (2021, 26 de octubre). Declaración final acerca de la novena reunión del Comité de Emergencia del Reglamento Sanitario Internacional (2005) sobre la pandemia de enfermedad por coronavirus (COVID-19). Recuperado de https://www.who.int/es/news/item/26-10-2021-statement-on-the-ninth-meeting-of-theinternational-health-regulations-(2005)-emergency-committee-regarding-the-coronavirusdisease-(covid-19)-pandemic

Patel, B. K., Wolfe, K. S., Pohlman, A.S., Hall, J.B. \& Kress, J.P. (2016). Effect of Noninvasive Ventilation Delivered by Helmet vs Face Mask on the Rate of Endotracheal Intubation in Patients with Acute Respiratory Distress Syndrome: A Randomized Clinical Trial. JAMA, 315(22), 2435-2441. doi:10.1001/jama.2016.6338

Pérez Sarmenti, I. (2020, 21 de agosto). En Argentina están probando un «casco» especial como sustituto a los respiradores. CNN en español. Recuperado de https://cnnespanol.cnn.com/video/coronavirus-argentina-respirador-no-invasivo-cascopkg-ivan-perez-sarmenti/

U.S. Food \& Drugs Administration. (2020). Expanded Access. Recuperado de https://www.fda.gov/news-events/public-health-focus/expanded-access 